\title{
Using exercise to protect physical and mental health in youth at risk for psychosis
}

\author{
Joseph Firth, ${ }^{1,2}$ Felipe Schuch, ${ }^{3}$ Vijay A. Mittal ${ }^{4,5}$ \\ ${ }^{1}$ Division of Psychology and Mental Health, University of Manchester, Manchester, UK; ${ }^{2}$ NICM Health Research Institute, Western \\ Sydney University, Westmead, Australia; ${ }^{3}$ Department of Sports Methods and Techniques, Federal University of Santa Maria, Santa \\ Maria, Brazil; ${ }^{4}$ Department of Psychology and Institute for Policy Research, Northwestern University, Evanston, IL; ${ }^{5}$ Department of \\ Psychiatry, Department of Medical Social Sciences, Institute for Innovations in Developmental Sciences, Northwestern University, \\ Chicago IL, USA
}

\begin{abstract}
A large body of literature has demonstrated that exercise interventions can improve a broad range of outcomes in people with established schizophrenia, including reducing psychiatric symptoms, increasing cognitive functioning, and improving physical health. Furthermore, these benefits seem just as pronounced in first-episode psychosis. However, there have been few clinical studies to date examining the effects of exercise in those found to be 'at-risk' of psychosis, particularly for those meeting the criteria for 'Clinical High Risk' (CHR) state (a classification which includes both those meeting the 'ultra-high risk for psychosis' criteria and/or those with 'atrisk mental states'). This is surprising, as a proportion of those in the CHR state go on to develop psychotic disorders, and a growing body of evidence suggests that early interventions in this period have significant potential to improve the course of illness. In this article, we shall review the existing literature for i) exercise as an adjunctive intervention for those treated for psychosis; ii) exercise as a standalone intervention in CHR groups; and iii) the rationale and supportive evidence for widescale use of exercise to preserve physical and mental health in those identified as at risk for psychosis. From this, we will put forth how the CHR phase represents an under-researched but highly-suitable timepoint for administering structured exercise interventions, in order to improve physical, psychological and neurocognitive outcomes; while also potentially reducing the odds of transition to full-threshold psychotic disorders. Following this, directions, recommendations and considerations around both the clinical implementation and future research around exercise in CHR individuals will be discussed.
\end{abstract}

Key words: Physical Activity; fitness; aerobic; psychotic disorders; youth mental health.

Correspondence: Joseph Firth, Division of Psychology and Mental Health, University of Manchester, Manchester, UK.

E-mail: joseph.firth@manchester.ac.uk

Citation: Firth, J., Schuch, F., \& Mittal V.A. (2020). Using exercise to protect physical and mental health in youth at risk for psychosis. Research in Psychotherapy: Psychopathology, Process and Outcome, 23(1), 33-37. doi: 10.4081/ripppo.2020.433

Acknowledgements: VM is supported by NIH R21/R33 Award MH103231. All authors declare no conflict of interests. JF was previously supported by a NICM-Blackmores Institute Fellowship and is currently supported by a University of Manchester Presidential Fellowship (P123958) and a UK Research and Innovation Future Leaders Fellowship (MR/T021780/1).

Received for publication: 15 October 2019.

Accepted for publication: 30 January 2020.

This work is licensed under a Creative Commons Attribution NonCommercial 4.0 License (CC BY-NC 4.0).

${ }^{\circ}$ Copyright: the Author(s), 2020

Licensee PAGEPress, Italy

Research in Psychotherapy:

Psychopathology, Process and Outcome 2020; 23:33-37

doi:10.4081/ripppo.2020.433

\section{Exercise in the Treatment of Mental Illness: An Overview}

Physical activity (i.e., any bodily movement produced by skeletal muscles that results in energy expenditure) and 'exercise' (i.e., any structured activity intended to maintain or improve physical fitness) are considered to be a central component of a healthy lifestyle (O'Donovan, Lee, Hamer, \& Stamatakis, 2017; Pedersen \& Saltin, 2015). Supporting this, there is a large body of research demonstrating that higher levels of physical activity are positively associated with reduced incidence of various non-communicable diseases, including diabetes, cardiovascular disease and cancer, along with improved life expectancy (O'Donovan et al, 2017; Pedersen \& Saltin, 2015). Along with the established physical health benefits, there is currently an increasing academic, clinical and public interest in using exercise interventions in the treatment of mental illness. Supporting this, recent metaanalyses of Randomized Controlled Trials (RCTs), which are considered the gold-standard of evidence in health intervention research, have produced compelling evidence for significant benefits of exercise interventions across a 
broad spectrum of mental disorders (Ashdown-Franks et al., 2019; Stubbs et al., 2018). In particular, numerous meta-analyses in people with depressive disorders (which are among the most common mental illnesses across the population) have shown that aerobic exercise is effective as a first-line treatment for moderate depression, and in the adjunctive treatment of major depressive disorder (Ashdown-Franks et al., 2019; Stubbs et al., 2018). Similarly, RCTs in individuals with attention-deficit and hyperactivity disorder, post-traumatic stress disorder, various anxiety disorders have all found significant benefits for mental health from exercise interventions (Ashdown-Franks et al., 2019).

Furthermore, an emerging body of research indicates that exercise can also be an efficacious adjunctive treatment for schizophrenia and related psychotic disorders, which are widely considered to be among the most severe mental health conditions. For example, a 2015 metaanalysis found that 90 minutes (or more) of structured exercise per week can significantly reduce both 'positive symptoms' (i.e. psychotic experiences relating to hearing voices, visual hallucinations, paranoia or aggression) and 'negative symptoms' (e.g. lack of affect, amotivation, and social withdrawal) in people with schizophrenia already receiving antipsychotic medications(Firth, Cotter, Elliott, French, \& Yung, 2015). Separately to the positive and negative symptoms which characterize the disorder, schizophrenia is also associated with severe 'cognitive deficits', i.e., impairments in concentration, memory and reasoning, which are not improved by antipsychotic medications. Promisingly, however, more recent evidence has confirmed that exercise interventions can also significantly improve cognition in people with schizophrenia, specifically through increasing performance in working memory, attention/vigilance, and social cognition domains (Firth et al., 2016). In light of this growing body of research, the recent European Psychiatric Association's guidance on physical activity for mental illness now recommends aerobic exercise interventions for managing residual symptoms in those treated for schizophrenia, and for improving cognitive functioning (Stubbs et al., 2018).

\section{The Existing Evidence for Physical Activity and Exercise as 'Early Interventions for Psychosis'}

While there is limited direct evidence from exercise studies in 'clinical high risk' (CHR) samples, accumulating findings from a variety of sources suggests considerable promise. First, investigators have observed that physical activity is a protective factor against incident psychosis in the general population (Brokmeier et al., 2020) .Further, data from the Northern Finland 1966 Birth Cohort demonstrate that adolescents (at age 14) with parental psychosis (i.e., the first generation of high-risk studies focused on those at familial or genetic high-risk) that were engaged at physical activity at least once every two days were $24 \%$ less likely to develop psychosis compared to those at equivalent genetic risk that performed physical activity less than once per week (Keskinen et al., 2018).

In addition to general population and genetic risk studies, we can draw some clues about efficacy of exercise treatments for younger and early psychosis individuals by examining the growing number of trials focusing on those in 'First-Episode Psychosis' (FEP; broadly defined as those within the first 5 years of psychotic disorders). For example, the IBEEP trial in 2015 examined the benefits of 10 weeks of combined aerobic and resistance training compared to treatment as usual in 38 young people with diagnoses of FEP. The intervention aimed to achieve at least 90 minutes per week of supervised, vigorous physical activity (Firth et al., 2016). Results showed moderately large and statistically significant reductions in total symptoms scores and negative symptom subscales from the exercise training compared to usual care. Notable improvements were also observed for various domains of cognitive functioning, particularly for verbal working memory, which remained statistically significant even after correcting for multiple testing $(\mathrm{p}=0.001)$. Similar results were found in a subsequent trial of aerobic exercise training in FEP; finding significant improvements in both global cognition after just 10 weeks, compared to usual care (Nuechterlein et al., 2016). Although both of these early studies used non-randomized designs, other recent studies, conducted as robust RCTs in FEP, have produced similar results: showing significant improvements in total symptoms, negative symptoms and working memory (along with other cognitive functions) from exercise, compared to a randomized waitlist control (Lin et al., 2015).

Despite these promising findings in FEP groups, it is important to remember that, in each of these studies, the participants were already receiving antipsychotic treatment for diagnosed psychotic disorders. Therefore, this literature currently only indicates that exercise can be used as an adjunctive intervention for people with FEP. However, the issue still remains as to whether or not exercise could be administered as an effective first-line treatment to antipsychotic-naïve in individuals, such as those displaying early indications of psychosis risk but prior to the onset of a psychotic disorder - in order to improve longterm outcomes and perhaps prevent the transition to 'full threshold' psychotic disorders in some cases.

The CHR criteria can be used as an evidence-based method for identifying young people at greatly elevated risk for developing a psychotic disorder within the next coming months or years (Yung et al., 2008). Meeting the criteria for CHR is determined by the presence of emergent or worsening attenuated positive symptoms, alongside a deteriorating in social and occupational functioning (Yung et al., 2008). Whereas CHR individuals typically have over a 20 -fold increase in risk of developing psychosis compared to the general population, timely intervention may reduce or delay the onset (van der Gaag et 
al., 2013). However, the evidence base for appropriate interventions during this time is patchy, as although antipsychotics can be effective for reducing attenuated positive symptoms (van der Gaag et al., 2013), this literature is inconsistent, and the risk of false positives (i.e., medicating those individuals who would not in fact be among the group that would eventually transition to psychosis) is a significant concern (Mittal, Dean, Mittal, \& Saks, 2015). In addition, the impact on negative symptoms and functioning is unknown, and side effects including weight gain and sedation impact adherence - and this must be balanced against the adverse effects on physical health (Mittal et al., 2015). Regarding non-pharmacological interventions, psychological therapies are potentially effective for CHR, but unfortunately are often costly, unavailable, or inaccessible for this non-diagnosed population (requiring highly specialized training, and typically provided only in major city centers). Further, the initial promise of other benign treatments (such as omega3 fish oils) has dissipated in light of null recent findings (Firth, 2019a). In addition, attrition for CHR individuals is a significant issue across treatment modalities, including those that require a heavy cognitive effort component or the need for traveling to a provider for multiple weekly sessions (Thompson et al., 2015). Furthermore, studies have further indicated that the high levels of sedentary behavior observed in CHR individuals is related to increased symptoms as well as poor brain health (Mittal et al., 2013). What is needed, to supplement these other approaches, or to serve as a primary approach, is a solution that is low cost, widely available, and self-reinforcing, that also has a favorable side-effect profile.

While there are just a handful of studies, the preliminary evidence also suggests that physical exercise may present a possible option, worthy of further research. Notably, a recent open-label study included 12 CHR individuals who engaged in 12-weeks of supervised aerobic exercise 2 to 3 times a week (Dean et al., 2017). The goal was to determine if exercise stimulated neurogenesis in the hippocampus, a region implicated in the pathogenesis of psychosis, and important for modulating cognitive function as well as psychosocial stress (Vargas et al., 2017). Participants were administered a comprehensive clinical, cognitive, fitness, and imaging battery pre and post the three month trial and during each session, exercised between $65-85 \%$ of Maximal Oxygen Capacity (MOC) for 30 minutes each session (because of the small number of participants, the investigators collapsed analyses across those receiving an easier [65\% MOC/2x per week] and more difficult [ $65 \% \mathrm{MOC} / 3 \mathrm{x}$ per week] exercise prescription assignments). The primary findings of the study indicated excellent tolerability as nine of the 12 individuals completed the trial. It is interesting, that the three who did drop out were each in the more difficult exercise prescription assignment and further, that the nine completers were very engaged, attending $100 \%$ of the sessions (depending on the assigned condition, this ranged from 24-36 separate exercise sessions). Findings revealed decreases in symptomatology post trial, with significant remission in both positive and negative symptom domains. The authors also observed increased social and role functioning post trial and further, increases in neurocognitive performance (i.e., working memory, verbal learning, visual learning, speed of processing, attention/vigilance, and reasoning and problem solving all improved while social cognition remained steady across the trial). In addition, the investigators reported normalization of functional connectivity between the left hippocampus and occipital cortex post trial. The same team of investigators moved to a second phase of this study to continue to examine the effectiveness of aerobic exercise in promoting brain health and improving related symptoms in a randomized controlled trial. The trial includes 15 CHR exercising, 15 CHR waitlist, and 15 healthy control participants; data collection is now completed and upcoming findings will be highly informative for ruling out demand characteristics, regression to the mean, and factors specific to psychosis risk individuals.

As noted, it is important to consider that despite the CHR criteria signifying much higher risk of psychosis than the very low rates observed across the general population, the majority of CHR individuals never go on to develop full-threshold psychotic disorders (Yung et al., 2008). Thus, it could be argued that in a world of limited resources, exercise interventions for psychosis would be better invested in diagnosed schizophrenia, or at least FEP; to ensure all those receiving the interventions fall within the target populations for experiencing tangible benefits. However, it must also be considered that even the CHR individuals who do not transition to schizophrenia are still at considerably higher risk of a plethora of psychiatric conditions, such as depression, anxiety and substance use disorders - all of which also often exist as a comorbidity during the CHR phase (Lin et al., 2015). Importantly, only a minority of CHR cases go onto a lifetime absence of mental illness (Lin et al., 2015). Given that physical activity has been shown to be effective in both the prevention and treatment of the broad range of other mental disorders(3), administering exercise interventions during the CHR phase has the clear potential of improving mental health outcomes in the vast majority of individuals, for both transitioning and non-transitioning cases. Indeed, a recent feasibility study of a 12weeks exercise intervention produced significant improvements in motivation, depression, and sleep problems in the 20 CHR participants - all aspects of the condition which are often left untreated (Lederman et al., 2019).

The rationale for implementing exercise within the standard care of CHR is further supported by the fact that along with mental illness, CHR individuals are also at significantly higher risk of chronic physical health conditions than the general population, due to an elevated rates of adverse health behaviors (such as smoking, inactivity, poor diet) observed in this population (Carney, Cotter, Bradshaw, Firth, 
$\&$ Yung, 2016). Whereas standard care may typically consist of psychological therapies addressing emergent symptoms, and pharmacological management of comorbid depression/anxiety, the addition of physical activity interventions could also reduce the risk of a number of noncommunicable conditions affecting those with CHR (O’Donovan et al., 2017; Pedersen \& Saltin, 2015). In light of this, the recent Lancet Psychiatry Commission (Firth, $2019 b$ ) recommends that physical activity is used as a core component of preventative health interventions from the very earliest stages of mental illness; in order to protect physical health from illness onset, and prevent the foreseeable chronic physical comorbidities (such as obesity, diabetes and cardiovascular diseases) from arising. Interestingly, although CHR populations have highly elevated behavioral risk factors, the incidence of cardiovascular and metabolic diseases among those with CHR is not much greater than the general population; likely due to the young age of this group meaning chronic conditions have yet to arise (Carney et al., 2016; Firth, 2019b). Thus, this presents an ideal timeframe for using physical activity (ideally within more broader lifestyle interventions) to address behavioral risks early, and avoid the clear path to cardiometabolic diseases along with the personal, social and economic burden these comorbidities bring (Firth, 2019b).

\section{Strategies and Considerations around Implementing Exercise in Youth Mental Health}

Regarding the implementation of physical activity interventions with CHR groups, the most optimal and costeffective methods have yet to be determined. Indeed, this is a fruitful and highly recommended area for further research. Nonetheless, there are some 'lessons learnt' from similar interventions in related populations, which can also be applied here.

First and foremost, exercise should be delivered flexibly, and customized to individual needs. Indeed, previous studies have found that the physical, psychological and cognitive benefits of physical activity can be obtained from various forms of exercise modalities; including aerobic fitness, resistance training, and sports groups (O'Donovan et al., 2017; Ashdown-Franks et al., 2019; Firth et al., 2015; Firth et al., 2016). Therefore, choosing types of activity which are amendable to patients, and even selected based on their preference, is the ideal strategy for promoting uptake and adherence to regular physical activity.

Second, wherever possible, exercise interventions for mental health should involve the input of 'physical activity professionals' i.e. individuals whose primary role (and training) is on the design and delivery of exercise training programs (e.g. physiotherapist, qualified fitness instructors, exercise physiologists). This is because mental health services are often among the most overstretched and under-resourced services within broader healthcare systems, and placing additional responsibilities (such as physical activity promotion) on existing mental health staff can impede the delivery of the necessary interventions, or even interfere with other important aspects of care. Alongside this, existing meta-analyses have convincingly demonstrated that exercise interventions for people with mental illness have substantially higher rates of adherence, lower drop-out, and better outcomes when delivered by exercise professionals, as opposed to researchers/mental health staff (Firth et al, 2016; Stubbs et al., 2016).

Third, considerations for the sustainability of exercise regimes should be considered from the outset, as research in other populations has indicated that the long-term benefits of exercise interventions are largely dependent on sustained adherence to regular physical activity (Pedersen \& Saltin, 2'15; Firth et al., 2018). Strategies for delivering exercise interventions with long-term sustainability include tying mental health services to community-based exercise programs, via the development of 'exercise on referral' schemes, and/or establishing permanent exercise facilities within mental health centers (Firth et al., 2019b). In this context it is important to consider that several studies have now observed that several barriers to exercise have been observed in CHR samples, including motivation as well as direct social interaction (Newberry, Dean, Sayyah, \& Mittal, 2018). Given these issues, emerging digital health technologies, such as smartphones, are likely to present promising new opportunities for integrating physical activity promotion within mental healthcare (Firth, 2019b). Overall however, while recent studies have demonstrated short-term benefits from exercise in CHR, further research is still required to establish the feasibility and effectiveness of using exercise for reserving mental health, and reducing risk of transition to psychosis, over extended periods of time.

\section{Conclusions}

Overall, the available evidence suggests exercise is a feasible and potentially effective intervention for young people at high risk for psychosis. Furthermore, implementing exercise during these early phases of illness appears to present an optimal timeframe for not only potentially delaying/preventing the onset of psychotic episodes, but also for providing young people with a self-management strategy for improving their psychological well-being, increasing cognitive functioning, and protecting their physical health. Currently however, there was been a lack of randomized controlled trials examining the efficacy of exercise specifically in CHR samples - with most of the suggested benefits implied from other populations with either firstepisode or established psychosis. However, initial trials in CHR does present preliminary evidence for physical exercise at this phase. Therefore, further research is urgently needed to i) establish the effects of clinically-implemented exercise interventions on physical and mental health outcomes in those at risk for psychosis; and ii) develop and disseminate pragmatic, cost-effective and sustainable meth- 
ods for delivering exercise interventions through relevant youth mental health services.

\section{References}

Ashdown-Franks G, Firth J, Carney R,Carvalho AF. Gallgren M, Koyanagi A, (...) Stubbs B. (2019). Exercise as medicine for mental and substance use disorders: a meta-review of the benefits for neuropsychiatric and cognitive outcomes. Sports Med 1-20. doi: 10.1007/s40279-019-01187-6.

Brokmeier L, Firth J, Vancampfort D, Smith L, Deenik J, (...) Schuch FB. (2020) Does physical activity reduce the risk of psychosis? A systematic review and meta-analysis of prospective studies. Psychiatry Res 2020;284:112675. doi: 10.1016/j.psychres.2019.112675.

Carney R, Cotter J, Bradshaw T, Firth J \& Yung AR. (2016). Cardiometabolic risk factors in young people at ultra-high risk for psychosis: A systematic review and meta-analysis. Schizophr Res 170:290-300. doi: 10.1016/j.schres.2016.01.010.

Dean DJ, Bryan AD, Newberry R, Gupta T, Carol E, Mittal VA (2017). A supervised exercise intervention for youth at risk for psychosis: an open-label pilot study. J Clin Psychiatry 78:e1167-e1173. doi: 10.4088/JCP.16m11365.

Firth J, Carney R, Elliott R, French P, Parker S, (...) Yung AR. (2016). Exercise as an intervention for first-episode psychosis: a feasibility study. Early Interv Psychiatry 12:30715. doi: 10.1111/eip.12329.

Firth J, Carney R, French P, Elliott R, Cotter J \& Yung AR. (2018). Long-term maintenance and effects of exercise in early psychosis. Early Interv Psychiatry 12:578-85. doi: 10.1111/eip.12365.

Firth J, Cotter J, Elliott R, French P, Yung AR. (2015). A systematic review and meta-analysis of exercise interventions in schizophrenia patients. Psychol Med 45:1343-61. doi:10.1017/S0033291714003110.

Firth J, Siddiqi N, Koyanagi A, Siskind D, Rosenbaum S, (...) Stubbs B. (2019b). The Lancet Psychiatry Commission: a blueprint for protecting physical health in people with mental illness. The Lancet Psychiatry 6:675-712.

Firth J, Stubbs B, Rosenbaum S, Vancampfort D, Machow B, (...) Yung AR. (2016). Aerobic exercise improves cognitive functioning in people with schizophrenia: a systematic review and meta-analysis. Schizophr Bull 43:546-56. doi: $10.1093 / \mathrm{schbul} / \mathrm{sbw} 115$.

Firth J, Teasdale S, Allot K, Siskind D, Marx W, Cotter J, (...) Sarris J. (2019a). The efficacy and safety of nutrient supplements in the treatment of mental disorders: a meta-review of meta-analyses of randomized controlled trials. World Psychiatry 18: doi: 10.1002/wps.20672.

Keskinen E, Marttila R, Koivumaa-Honkanen H, Moilanen K, Keinänen-Kiukaanniemi S. (...) Jääskeläinen E. (2018). Search for protective factors for psychosis-a populationbased sample with special interest in unaffected individuals with parental psychosis. Early Interv Psychiatry 12:869-78. doi: 10.1111/eip.12380.

Lin A, Wood SJ, Nelson B, Beavan B, McGorry P, Yung AR. (2015). Outcomes of nontransitioned cases in a sample at ultra-high risk for psychosis. Am J Psychiatry 172:249-58, doi: 10.1176/appi.ajp.2014.13030418.

Lederman O, Ward PB, Rosenbaum S, Maloney C, Watkins, (... ) Curtis J. (2019). Stepping up early treatment for help-seek- ing youth with at-risk mental states: Feasibility and acceptability of a real-world exercise program. Early Interv Psychiatry doi: 10.1111/eip.12871.

Lin J, Chan SKW, Lee EH, Chang WC, Tse M, (...) Chen EYH. (2015). Aerobic exercise and yoga improve neurocognitive function in women with early psychosis. NPJ Schizophr 1:15047. doi: $10.1038 /$ npjschz.2015.47.

Mittal VA, Dean DJ, Mittal J, Saks E.R. (2015). Ethical, legal, and clinical considerations when disclosing a high-risk syndrome for psychosis. Bioethics 29:543-56. doi: 10.1111/bioe.12155.

Mittal VA, Gupta T, Orr JM, Pelletier-Baldelli A, Dean DJ, (...) Millman ZB. (2013). Physical activity level and medial temporal health in youth at ultra-high-risk for psychosis. J Abnormal Psychol 122:1101-10. doi: 10.1037/a0034085.

Newberry RE, Dean DJ, Sayyah MD, Mittal VA. (2018). What prevents youth at clinical high risk for psychosis from engaging in physical activity? An examination of the barriers to physical activity. Schizoph Res 201:400-5. doi: 10.1016/j.schres.2018.06.011.

Nuechterlein KH, Ventura J, McEwen SC, Gretchen-Doorly D, Vinogradov S, Subotnik KL. (2016). Enhancing cognitive training through aerobic exercise after a first schizophrenia episode: theoretical conception and pilot study. Schizophr Bull 42:S44-S52. doi: 10.1093/schbul/sbw007.

O'Donovan G, Lee IM, Hamer M, Stamatakis E. (2017). Association of "Weekend Warrior" and Other Leisure Time Physical Activity Patterns with Risks for All-Cause, Cardiovascular Disease, and Cancer Mortality. JAMA Intern Med 177:335-42. doi: 10.1001/jamainternmed.2016.8014.

Pedersen BK, Saltin B. (2015) Exercise as medicine-evidence for prescribing exercise as therapy in 26 different chronic diseases. Scand J Med Sci Sports 25:1-72. doi: 10.1111/sms.12581.

Stubbs B, Vancampfort D, Hallgren M, Firth J, Veronese N, Solmi M, (...) Kahl KG. (2018). EPA guidance on physical activity as a treatment for severe mental illness: a meta-review of the evidence and Position Statement from the European Psychiatric Association (EPA), supported by the International Organization of Physical Therapists in Mental Health (IOPTMH). Eur Psychiatry 54:124-44. doi: 10.1016/j.eurpsy.2018.07.004.

Stubbs B, Vancampfort D, Rosenbaum S, Ward PB, Richards J, (...) Schuch FB. (2016). Dropout from exercise randomized controlled trials among people with depression: a metaanalysis and meta regression. J Affect Disord 190:457-66. doi: 10.1016/j.jad.2015.10.019.

Thompson E, Millman ZB, Okuzawa N, Mittal V, DeVylder J, Skadberg T, (...) Schiffman J. (2015). Evidence-based early interventions for individuals at clinical high risk for psychosis: a review of treatment components. J Nervous Mental Dis 203:342-51. doi: 10.1097/NMD.0000000000000287.

van der Gaag M, Smit F, Bechdolf A, French P, Linszen DH, (... ) Cuijpers P. (2013). Preventing a first episode of psychosis: Meta-analysis of randomized controlled prevention trials of 12 month and longer-term follow-ups. Schizophr Res 149:56-62. doi: 10.1016/j.schres.2013.07.004.

Vargas T, Dean DJ, Osborne KJ, Gupta T, Ristanovic I, (...) Mittal VA. (2017). Hippocampal subregions across the psychosis spectrum. Schizophr Bull 44:1091-9. doi: 10.1093/ schbul/sbx 160 .

Yung AR, Nelson B, Stanford C, Simmons MB, Cosgrave EM, Killackey E, (...) McGorry PD. (2008). Validation of "prodromal" criteria to detect individuals at ultra-high risk of psychosis: 2 year follow-up. Schizophr Res 105:10-7. doi: 10.1016/j.schres.2008.07.012. 